\title{
Multiscale Chebyshev Neural Network Identification and Adaptive Control for Backlash-Like Hysteresis System
}

\author{
Xuehui Gao $(\mathbb{D})$ and Ruiguo Liu (iD \\ Department of Mechanical and Electrical Engineering, Shandong University of Science and Technology, Tai'an 271019, China \\ Correspondence should be addressed to Ruiguo Liu; lrgsdkd@163.com
}

Received 18 May 2018; Accepted 14 August 2018; Published 3 October 2018

Academic Editor: Zhile Yang

Copyright ( 2018 Xuehui Gao and Ruiguo Liu. This is an open access article distributed under the Creative Commons Attribution License, which permits unrestricted use, distribution, and reproduction in any medium, provided the original work is properly cited.

\begin{abstract}
An adaptive control based on a new Multiscale Chebyshev Neural Network (MSCNN) identification is proposed for the backlashlike hysteresis nonlinearity system in this paper. Firstly, a MSCNN is introduced to approximate the backlash-like nonlinearity of the system, and then, the Lyapunov theorem assures the identification approach is effective. Afterward, to simplify the control design, tracking error is transformed into a scalar error with Laplace transformation. Therefore, an adaptive control strategy based on the transformed scalar error is proposed, and all the signals of the closed-loop system are uniformly ultimately bounded (UUB). Finally, simulation results have demonstrated the performance of the proposed control scheme.
\end{abstract}

\section{Introduction}

As one of the typical nonlinearity, hysteresis exists in many intelligent materials, servo motor systems, mechanical systems, and others [1-3], and it has attracted much interest for many years. Many hysteresis models have been investigated including mathematical model and physical model. For these models, Preisach model [1, 4], Prandtl-Ishlinskii (P-I) model [5, 6], Bouc-Wen model [7, 8], and backlashlike model $[2,3]$ are important in hysteresis research.

Backlash-like model has been firstly investigated for hysteresis systems with a differential equation in [2]. A robust adaptive control strategy was designed without constructing inverse hysteresis nonlinearity in [2]. Zhou et al. [3] have utilized backstepping technique designing a robust adaptive backstepping control to deal with the unknown backlashlike hysteresis nonlinearity with bounded external disturbances. H. J. Lee and J. J. Lee [9] applied the results for Shape memory alloy (SMA) actuators, and a control strategy was adopted for the time delay control of the backlash-like hysteresis system.

The investigation of backlash-like hysteresis model has attracted attention for these years. Compared with the backstepping technique, sliding mode control has less calculated amount. Zhang et al. [10] have designed a sliding mode control for the backlash-like hysteresis system based on Ackermann's formula. Different from the above literatures, Dong et al. [11] investigated nonsmooth predictive control backlash-like hysteresis mechanical transmission systems. The backlash-like model was described by a nonsmooth function, and a model predictive control was presented with a nonsmooth receding horizon strategy. Liu et al. [12] have investigated multiple-input multiple-output (MIMO) systems with backlash-like hysteresis model and proposed an adaptive prescribed performance control to precision control the system. More and more researchers pay great attentions to the prescribed performance control [13-20] in recent years. But prescribed performance control for hysteresis system investigation needs more attention from researchers.

Chebyshev neural network (CNN) is one of the functional-link neural network (FLNN) which is only one layer and omit the hidden layer compared with other multineural networks. Therefore, CNN has lower computation for its simple structure. The CNN has been widely utilized in permanent magnet synchronous motor (PMSM) system $[21,22]$, spacecraft system, and others $[23,24]$. Zou et al. [23] proposed a terminal sliding mode control (TSMC) and 
CNN for spacecraft. The attitude and velocity error have been transformed into a double integrator for the spacecraft system, and then, a switch function was adopted to generate a switching between the adaptive $\mathrm{NN}$ and the robust controller. An adaptive TSMC-based CNN was proposed for tracking the attitude of the spacecraft. Different from [23], Lin [21] investigated a hybrid recurrent CNN for the PMSM of the continuously variable transmission (CVT) system. The gradient descent method of the adaptive law in the HRCNN was introduced, and the stability was assured by Lyapunov function. Sun et al. [22] adopted a modified dynamic surface control with high-order sliding mode (HOSM) differentiator approximating the unknown velocity of the multimotor servomechanism. The unknown friction and disturbance were estimated by CNN. Above literatures all investigated system control with CNN but not applied to system identification. Patra and Kot [25] firstly proposed CNN to identify the dynamic nonlinear system where it needed less computation. As a whole, system identification with $\mathrm{CNN}$ has few research for the researchers. Meanwhile, adaptive control [26-29] is an extensive control strategy that has been investigated for many years. In this paper, an adaptive CNN controller will be investigated for the backlash-like hysteresis nonlinear system.

Multiscale identification was adopted in [30,31] for singularly perturbed nonlinear system with multitime-scale recurrent high-order neural networks (MSRHONN). A new optimal bounded ellipsoid algorithm was applied for MSRHONN which had faster convergence due to the adaptively adjusted learning rate. Different from Zheng et al. $[30,31]$, we will propose a CNN to identify the backlashlike hysteresis system and design an adaptive control strategy for this system. Firstly, the CNN identification will be designed and guaranteed by Lyapunov function with update law. Then, the tracking error will be transformed into scalar form through Laplace transportation to simplify the controller design. Finally, an adaptive neural network will be proposed for the backlash-like hysteresis nonlinearity system and assured by Lyapunov theorem.

\section{Problem Formulation}

The backlash-like hysteresis system of this paper is described as follows:

$$
\left\{\begin{array}{l}
\dot{x}=f_{1}(x)+f_{2}(x) v, \\
\dot{v}=f_{3}(v, u),
\end{array}\right.
$$

where $f_{1}, f_{2}$ are unknown nonlinear smooth functions, and $x$, $v$ have different time scale; $v$ represents hysteresis nonlinearity; it is formulated by nonlinear smooth function $f_{3}$; in this paper, the function $f_{3}$ is defined by backlash-like model, i.e., $f_{3}$ is described by the following equation:

$$
\dot{v}=f_{3}(v, u)=\alpha|\dot{u}|(\beta u-v)+\gamma \dot{u},
$$

where $\alpha$ is positive coefficient, and $u$ means the input signal of the nonlinear system; $\beta, \gamma$ are constants of the hysteresis nonlinearity, and $\beta>0$ satisfying $\beta>\gamma$.

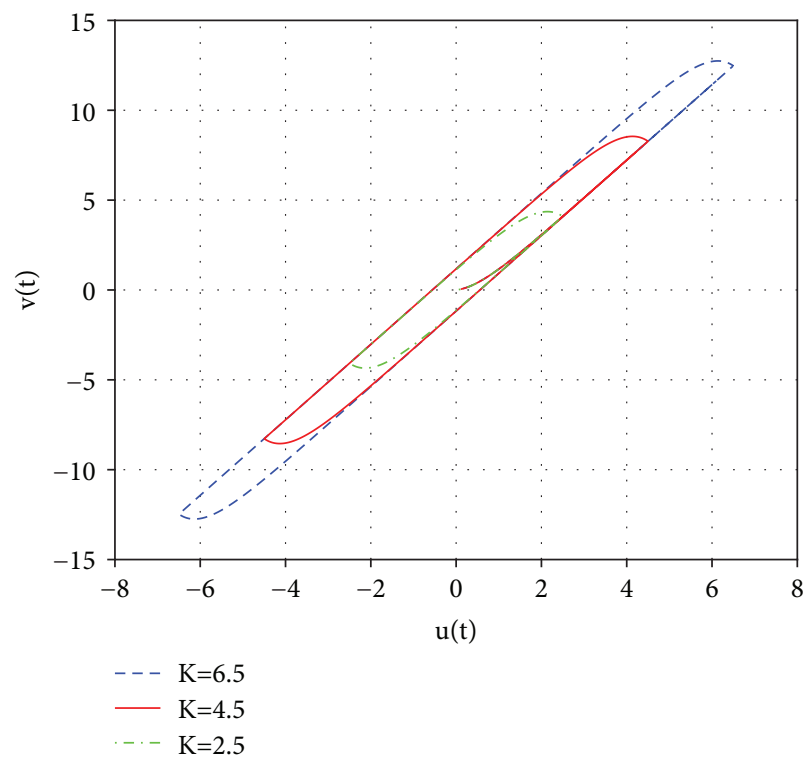

FIGURE 1: The curve of the backlash-like hysteresis model.

TABle 1: The backlash-like model parameters.

\begin{tabular}{lcccccc}
\hline Backlash-like parameters & $\alpha$ & $\beta$ & $\gamma$ & $K_{1}$ & $K_{2}$ & $K_{3}$ \\
Value & 1.6 & 0.22 & 2.1 & 6.5 & 4.5 & 2.5 \\
\hline
\end{tabular}

According to the literature [2], (2) is solved as follows:

$$
v=\beta u+\Xi(u),
$$

where

$$
\begin{aligned}
\Xi(u)= & \left(v_{0}-\beta u_{0}\right) e^{-\alpha\left(\left(u-u_{0}\right) \operatorname{sign}(u)\right)} \\
& +e^{-\alpha u \operatorname{sign}(u)} \int_{u_{0}}^{u}(\gamma-\beta) e^{\alpha \zeta \operatorname{sign}(u)} d \zeta .
\end{aligned}
$$

The backlash-like hysteresis curves are illustrated in Figure 1 where the model parameters are listed in Table 1. The input signals of the backlash-like hysteresis model are selected as $u(t)=K_{i} \sin (2.5 t), i=1,2,3$, respectively; $K_{i}$ is chosen in Table 1.

\section{Multiscale Neural Network Identification Design}

In order to identify the hysteresis system (1), a multiscale CNN is introduced and then is applied for the backlash-like hysteresis nonlinearity to approximate the system.

3.1. Chebyshev Neural Network. The CNN is one kind of FLN based on Chebyshev polynomials actually which is only single-layer and omits hidden layer compared with other neural network such as Multilayer Neural Network (MNN). $\mathrm{CNN}$ structure includes two sections: Chebyshev expansion block and learning block. A structure of $\mathrm{CNN}$ is shown in 


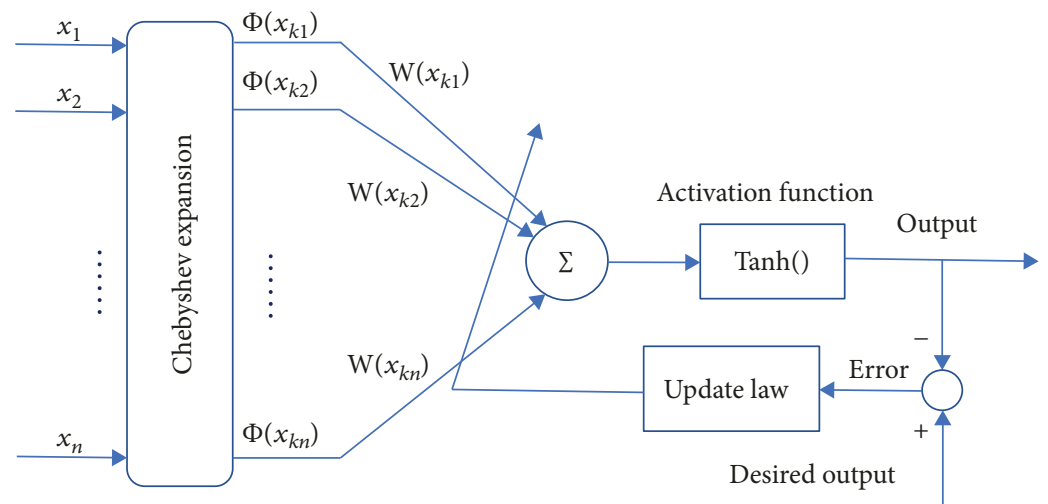

FIGURE 2: The Chebyshev neural network structure.

Figure 2 in this paper. Then, the $\mathrm{CNN}$ is defined as

$$
F(x)=W^{*} \Phi(x)+\varepsilon,
$$

where $W^{*} \in \mathbb{R}^{n \times n}$ means the weight matrix of the $\mathrm{CNN}$, and $\varepsilon$ denotes the bounded approximation error of the $\mathrm{CNN}$.

$$
\begin{aligned}
\Phi(x)= & {\left[1, C_{1}\left(x_{1}\right), \cdots, C_{n}\left(x_{1}\right), C_{1}\left(x_{2}\right), \cdots, C_{n}\left(x_{2}\right),\right.} \\
& \left.\cdots, C_{1}\left(x_{m}\right), \cdots, C_{n}\left(x_{m}\right)\right]^{T},
\end{aligned}
$$

and $\left[x_{1}, x_{2}, \cdots, x_{m}\right]$ is the $m$-dimensional input vector; $n$ denotes the order of the Chebyshev polynomial; the $k+1$ th order Chebyshev polynomial $C_{k+1}(x)$ is selected as the wellknown two-term recursive formula:

$$
C_{k+1}(x)=2 x C_{k}(x)-C_{k-1}(x),
$$

where $C_{1}(x)=x, C_{0}(x)=1$.

3.2. Multiscale Identification. Considering the hysteresis system (1) with backlash-like hysteresis model (2), the new CNNs are utilized to estimate the system as follows:

$$
\dot{\hat{x}}=A \widehat{x}+\widehat{W}_{1} \Phi_{1}(x)+\widehat{W}_{2} \Phi(x) v
$$

where $\hat{x}$ is the estimation of the hysteresis system states; $A$ is the designed diagonal stable matrix; $\widehat{W}_{1} \in \mathbb{R}^{n \times n}, \widehat{W}_{2} \in \mathbb{R}^{n \times n}$ are the weights of the CNN.

The backlash-like hysteresis nonlinearity system can be approximated by the optimal $\mathrm{CNN}$ as

$$
\dot{x}=A x+W_{1}^{*} \Phi_{1}(x)+W_{2}^{*} \Phi_{2}(x) v+\varepsilon,
$$

where $\varepsilon$ represents the modeling error.

Define the error $e$ as

$$
e=x-\widehat{x}
$$

Then, according to (8) and (9), the derivative of $e$ can be deduced as

$$
\dot{e}=\dot{x}-\dot{\hat{x}}=A e+\left(W_{1}^{*}-\widehat{W}_{1}\right) \Phi_{1}(x)+\left(W_{2}^{*}-\widehat{W}_{2}\right) \Phi_{2}(x) v+\varepsilon .
$$

Defining $\tilde{W}_{1}=W_{1}^{*}-\widehat{W}_{1}, \tilde{W}_{2}=W_{2}^{*}-\widehat{W}_{1}$, (11) can be rewritten as

$$
\dot{e}=A e+\tilde{W}_{1} \Phi_{1}(x)+\tilde{W}_{2} \Phi_{2}(x) v+\varepsilon
$$

The following assumptions are desired for the identification of the backlash-like hysteresis system:

Assumption 1. The CNN modeling approximation error $\varepsilon$ is bounded such that $|\varepsilon| \leq \varepsilon_{M}$, where $\varepsilon_{M}$ is a known positive bound.

Assumption 2. The optimal CNN weight matrix $W_{i}^{*}, i=1,2$, is bounded, such that

$$
\begin{aligned}
& \operatorname{tr}\left\{W_{1}^{* T} W_{1}^{*}\right\} \leq W_{1 M}, \\
& \operatorname{tr}\left\{W_{2}^{* T} W_{2}^{*}\right\} \leq W_{2 M},
\end{aligned}
$$

where $W_{1 M}, W_{2 M}$ are known positive constants, respectively.

For the system (9) with diagonal stable matrix $A$, the following equation holds:

$$
A^{T} P+P A \leq-Q
$$

where $Q$ is a positive definite symmetric matrix.

The update law of CNN can be designed as

$$
\begin{aligned}
& \dot{\widehat{W}}_{1}=\delta_{1} e^{T} P \Phi(x)-\delta_{1} \delta_{2} \widehat{W}_{1}, \\
& \dot{\widehat{W}}_{2}=\delta_{3} e^{T} P \Phi(x) v-\delta_{3} \delta_{4} \widehat{W}_{2},
\end{aligned}
$$

where $\delta_{i}, i=1,2,3,4$, are designed positive constants, respectively. 
Next, the identification of the backlash-like hysteresis system will be guaranteed by Lyapunov theorem; a Lyapunov function is selected as

$$
V_{0}=\frac{1}{2} e^{T} P e+\frac{1}{2 \delta_{1}} \operatorname{tr}\left\{\tilde{W}_{1}^{T} \tilde{W}_{1}\right\}+\frac{1}{2 \delta_{3}} \operatorname{tr}\left\{\tilde{W}_{2}^{T} \tilde{W}_{2}\right\}
$$

Considering Assumption 1 and Assumption 2, (12), (14), and the CNN update law (15), the derivative of $V_{0}$ is deduced as

$$
\begin{aligned}
\dot{V}_{0} & =\frac{1}{2}\left(\dot{e}^{T} P e+e^{T} p \dot{e}\right)-\frac{1}{2 \delta_{1}} \operatorname{tr}\left\{\tilde{W}_{1}^{T} \dot{\hat{W}}_{1}\right\}-\frac{1}{2 \delta_{3}} \operatorname{tr}\left\{\tilde{W}_{2}^{T} \dot{\hat{W}}_{2}\right\} \\
& \leq-\frac{1}{2} e^{T} Q e-\varepsilon+\frac{\delta_{2}}{2} \operatorname{tr}\left\{\tilde{W}_{1}^{T} \widehat{W}_{1}\right\}+\frac{\delta_{4}}{2} \operatorname{tr}\left\{\tilde{W}_{2} \widehat{W}_{2}\right\} \\
& \leq-\frac{1}{2} e^{T} Q e+\varepsilon_{M}+\frac{\delta_{2} W_{1 M}}{2}+\frac{\delta_{4} W_{2 M}}{2},
\end{aligned}
$$

where the fact that

$$
\begin{aligned}
& \frac{\delta_{2}}{2} \operatorname{tr}\left\{\tilde{W}_{1}^{T} \widehat{W}_{1}\right\} \leq \frac{\delta_{2} W_{1 M}}{2}, \\
& \frac{\delta_{4}}{2} \operatorname{tr}\left\{\tilde{W}_{2} \widehat{W}_{2}\right\} \leq \frac{\delta_{4} W_{2 M}}{2}
\end{aligned}
$$

are utilized, and $c_{1}=\varepsilon_{M}+\left(\delta_{2} W_{1 M} / 2\right)+\left(\delta_{4} W_{2 M} / 2\right)$; thus, $\dot{V}_{0}$ satisfies the following inequality:

$$
\dot{V}_{0} \leq-\rho_{1} V_{0}+\rho_{2}
$$

where $\rho_{1}>0, \rho_{2}>0$.

Therefore, the error $e$ can be solved as

$$
\|e\| \leq \sqrt{2\left(\frac{\rho_{2}}{\rho_{1}}+V_{0}(0)\right) e^{-\rho_{1} t}}
$$

Considering $t \rightarrow \infty$, we have

$$
\|e\|_{t \rightarrow \infty} \leq \lim _{t \rightarrow \infty} \sqrt{2\left(\frac{\rho_{2}}{\rho_{1}}+V_{0}(0)\right) e^{-\rho_{1} t}}=\sqrt{2 \frac{\rho_{2}}{\rho_{1}}} .
$$

Then, the proposed CNNs can identify the backlash-like hysteresis nonlinearity system (1), and all the signals of the closed-loop are UUB. Next, an adaptive controller will be designed to control the system, and the Lyapunov theorem will guarantee the UUB of the closed-loop system.

Remark 1. CNN is a FLNN consists of two parts: the numerical transformation and learning. It has been proven that $\mathrm{CNN}$ has stronger approximation capabilities and has less calculate quantities since it is a single-layer neural network based on Chebyshev polynomials. In this paper, we designed two CNNs to estimate the unknown system parameters where the two CNNs have different scales. The multiscale $\mathrm{CNN}$ can be separated into the different unknown nonlinear dynamics where it will reduce the system order and simplify the structure of the controller.

\section{Adaptive Controller Design}

4.1. Error Transformation. The control objective of this paper is to design an adaptive controller for the backlash-like hysteresis nonlinearity system such that the tracking follows the reference model as

$$
\dot{x}_{r}=A_{r} x_{r}+b_{r} u_{r}
$$

where $A_{r}, B_{r}$ are known controllable state coefficient matrices, and $u_{r}$ represents the reference input signal.

In order to simplify the controller design, the following assumption and lemma are introduced.

Assumption 3. There exist positive constant vector $\vartheta_{x}^{*}$ and known nonzero constant $\vartheta_{r}^{*}$ such that the following equations are satisfied:

$$
\left\{\begin{array}{l}
A_{r}=A+B \vartheta_{x}^{*}, \\
b_{r}=B \vartheta_{r}^{*} .
\end{array}\right.
$$

Then, the following lemma exists:

Lemma 1 [32]. Let

$$
\left\{\begin{array}{l}
\dot{x}=A_{m} x+b m, \\
\lambda(s)=(s+r) H(s),
\end{array}\right.
$$

where $A_{m}$ is a diagonal stable matrix; $\lambda(s)$ denotes characteristic polynomial of $A_{m} ; r$ represents a designed positive constant; $H(s)$ is Hurwitz, and $A_{m}, b$ are controllable. Then, $\exists \eta$ such that

$$
\eta^{T}\left(s I-A_{m}\right)^{-1} b=\frac{1}{s+r} .
$$

The tracking error is defined as

$$
e_{t}=x-x_{r}
$$

In view of (3), (9), (22), and (23), the derivative of $e_{t}$ can be expressed as

$$
\begin{aligned}
\dot{e}_{t}= & \dot{x}-\dot{x}_{r} \\
= & A_{r} e_{t}+b_{r}\left(u_{r}-\frac{\vartheta_{x}^{*}}{\vartheta_{r}^{*}} x\right)+W_{1}^{*} \Phi_{1}(x) \\
& +W_{2}^{*} \Phi_{2}(x)(\beta u+\Xi(u))+\varepsilon .
\end{aligned}
$$

The Laplace transformation is utilized for the derivative of the tracking error $e_{t}$ in (27) as 


$$
\begin{aligned}
e_{t}(s)= & \left(s I-A_{r}\right)^{-1} b_{r}\left(u_{r}(s)-\frac{\vartheta_{x}^{*}}{\vartheta_{r}^{*}} x(s)\right)+W_{1}^{*} \Phi_{1}(x(s)) \\
& +W_{2}^{*} \Phi_{2}(x(s))(\beta u(s)+\Xi(u(s)))+\varepsilon(s) .
\end{aligned}
$$

Defining $e_{s}=\eta^{T} e_{t}$, considering (25), both sides of (28) multiply $\eta^{T}$, and then utilize inverse Laplace transformation, one has

$\dot{e}_{s}=-r e_{s}+\left(\vartheta_{r}^{*} u_{r}-\vartheta_{x}^{*} x\right)+\frac{W_{1}^{*} \Phi_{1}(x)+W_{2}^{*} \Phi_{2}(x)(\beta u+\Xi(u))+\varepsilon}{b_{r}}$.

4.2. Adaptive Controller Design. In order to design an adaptive controller for the backlash-like hysteresis nonlinearity system, the following variables are firstly defined as

$$
\left\{\begin{array}{l}
\tilde{\vartheta}_{x}=9_{x}^{*}-\widehat{\vartheta}_{x}, \\
\tilde{\vartheta}_{r}=\vartheta_{y}^{*}-\widehat{\vartheta}_{r},
\end{array}\right.
$$

where $\widehat{\vartheta}_{x}, \widehat{\vartheta}_{r}$ are the estimation of $\vartheta_{x}^{*}, \vartheta_{r}^{*}$, respectively.

The controller is designed as

$$
u=\frac{b_{r}}{\widehat{W}_{2} \Phi_{2}(x) \beta}\left(\widehat{\vartheta}_{x} \widehat{x}-\widehat{\vartheta}_{r} u_{r}\right)-\frac{\widehat{W}_{1} \Phi_{1}(x)-\Xi(u)}{\widehat{W}_{2} \Phi_{2}(x) \beta},
$$

and the adaptive law is selected as

$$
\dot{\widehat{\vartheta}}_{r}=\delta_{5} u_{r},
$$

where $\delta_{5}>0$ is designed constants.

Then, the following theorem holds.

Theorem 1. The backlash-like hysteresis nonlinearity system is described as (1), which is approximated by CNN in (8); the reference model is selected as (22); the controller is designed as (31), and the adaptive law is selected as (32). Then, the tracking error and other signals in closed-loop are UUB.

Proof 1. Select a Lyapunov function as

$$
V=\frac{1}{2} e_{s}^{2}+\frac{1}{2 \delta_{5}} \tilde{\vartheta}_{r}^{2} .
$$

Considering (29) and (30), the derivative of $V$ can be deduced as

$$
\begin{aligned}
\dot{V}= & e_{s} \dot{e}_{s}+\frac{1}{\delta_{5}}\left(\tilde{\vartheta}_{r} \dot{\tilde{\vartheta}}_{r}\right) \\
= & e_{s}\left(-r e_{s}+\left(\vartheta_{r}^{*} u_{r}-\vartheta_{x}^{*} x\right)+\frac{W_{1}^{*} \Phi_{1}(x)+W_{2}^{*} \Phi_{2}(x)(\beta u+\Xi(u)+\varepsilon)}{b_{r}}\right) \\
& +\frac{1}{\delta_{5}}\left(\tilde{\vartheta}_{r} \dot{\tilde{\vartheta}}_{r}\right) .
\end{aligned}
$$

Substituting (31) and (32) into (34), one has

$$
\begin{aligned}
V= & -r e_{s}^{2}+e_{s}\left(\vartheta_{r}^{*} u_{r}-\vartheta_{x}^{*} x+\frac{W_{1}^{*} \Phi_{1}(x)}{b_{r}}-\widehat{\vartheta}_{r} u_{r}+\widehat{\vartheta}_{x} \widehat{x}\right. \\
& \left.-\frac{\widehat{W}_{1} \Phi_{1}(x)+\Xi(u)+\varepsilon}{b_{r}}\right)+\frac{1}{\delta_{5}}\left(\tilde{\vartheta}_{r} \dot{\tilde{\vartheta}}_{r}\right) \\
\leq & -r e_{s}^{2}+e_{s}\left(\frac{\tilde{W}_{1} \Phi_{1}(x)}{b_{r}}-\tilde{\vartheta}_{x} e+\frac{\tilde{W}_{2} \Phi_{2}(x)}{\widehat{W}_{2} \Phi_{2}(x)}+\frac{\varepsilon}{b_{r}}\right) \\
\leq & -r e_{s}^{2}+\left|e_{s}\right|\left(\left|\frac{W_{1 M}}{b_{r}}\right|-\rho \sqrt{2 \frac{\rho_{2}}{\rho_{1}}}+|\varsigma|+\left|\frac{\varepsilon_{M}}{b_{r}}\right|\right) .
\end{aligned}
$$

Therefore, we have

$$
\dot{V} \leq-\psi_{1} V+\psi_{2}
$$

Then, the error $e_{s}$ is deduced as

$$
\left|e_{s}\right| \leq \sqrt{2\left(\frac{\psi_{2}}{\psi_{1}}+V(0)\right) e^{-\psi_{1} t}}
$$

According to Lyapunov theorem, the error $e_{s}$ is bounded and converged to a compact set, and others signals are all bounded.

Remark 2. The proposed approach can be tracked in the reference model through the transformed tracking scalar error under Assumptions 1, 2, and Assumption 3. For CNN, Assumption 1 and Assumption 2 require that the approximation error $\varepsilon$ and the weight matrix $W$ are bounded. It suits the real condition since the weight cannot select infinity. Assumption 3 and Lemma 1 can transform the vector error into a scalar error to simplify the controller design but degrade the control precision in fact.

\section{Simulations}

To verify the effectiveness of the CNN identification and adaptive control, the following system is considered:

$$
\left\{\begin{array}{l}
\dot{x}_{1}=x_{2}, \\
\dot{x}_{2}=g_{1}(x)+g_{2}(x) v, \\
\dot{v}=1.4|\dot{u}|(4.2 u-v)+0.38 \dot{u},
\end{array}\right.
$$

where $g_{1}(x)=0.5 \sin \left(x_{1}\right) x_{2}^{2}+1.8 \cos \left(x_{1}\right), g_{2}(x)=0.2 \cos$ $\left(x_{1}\right)+1, \alpha=1.4, \beta=4.2, \gamma=0.38$, and $u=2 \sin (2 t)$.

The reference model is selected as

$$
\dot{x}_{r}=A_{r} x_{r}+b_{r} u_{r},
$$



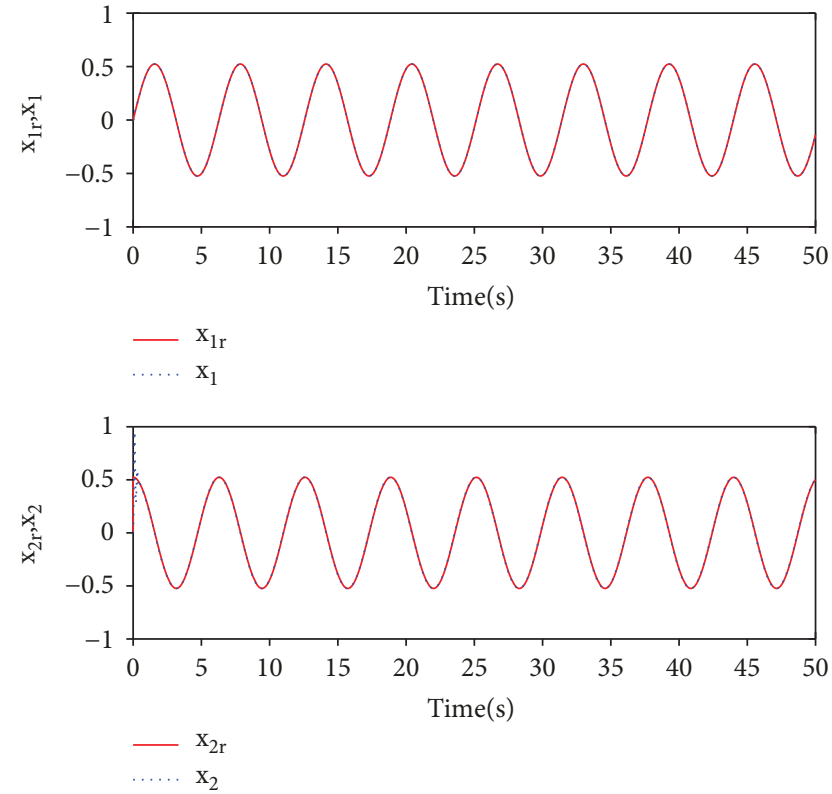

FIGURE 3: The adaptive control results of $x$ versus $x_{r}$ for $u_{r}=(\pi / 6)$ $\sin (t)$.
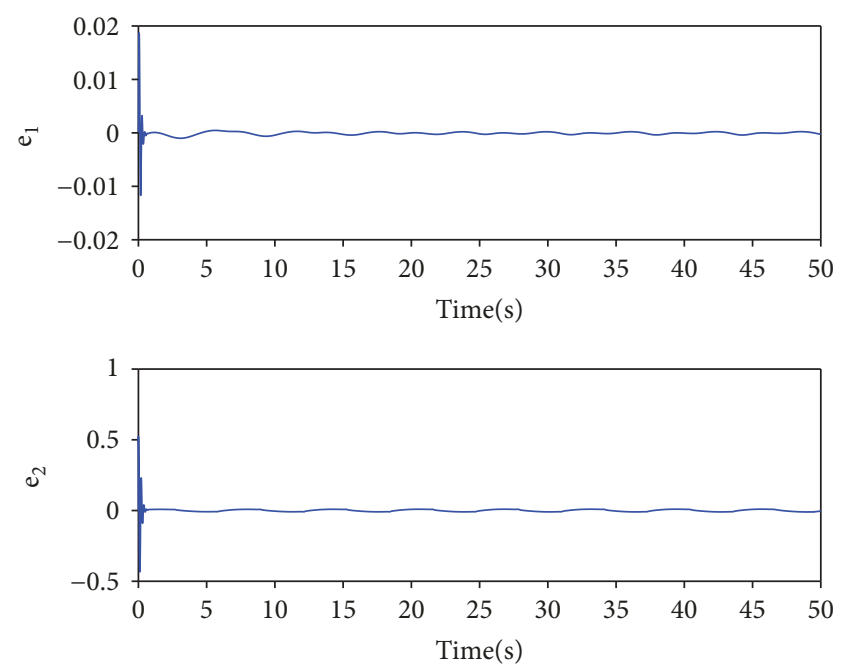

FIgURE 4: The errors of adaptive controller for $u_{r}=(\pi / 6) \sin (t)$.

where

$$
A_{r}=\left[\begin{array}{cc}
0 & 1 \\
-2 & -3
\end{array}\right] \text {, }
$$

and $b_{r}=3$.

The parameters of the $\mathrm{CNN}$ and adaptive law are chosen; $W_{1}(0)=0, W_{2}(0)=0, \varepsilon_{m}=0.05, \delta_{1}=100, \delta_{2}=0.002, \delta_{3}=$ $150, \delta_{4}=0.001, \delta_{5}=0.15$, and $r=3$. Figures $3-5$ illustrate the proposed adaptive control results in this paper. Figure 3 shows the adaptive control results versus the reference model; Figure 4 shows the tracking errors of the proposed approaches, and Figure 5 illustrates the control input.

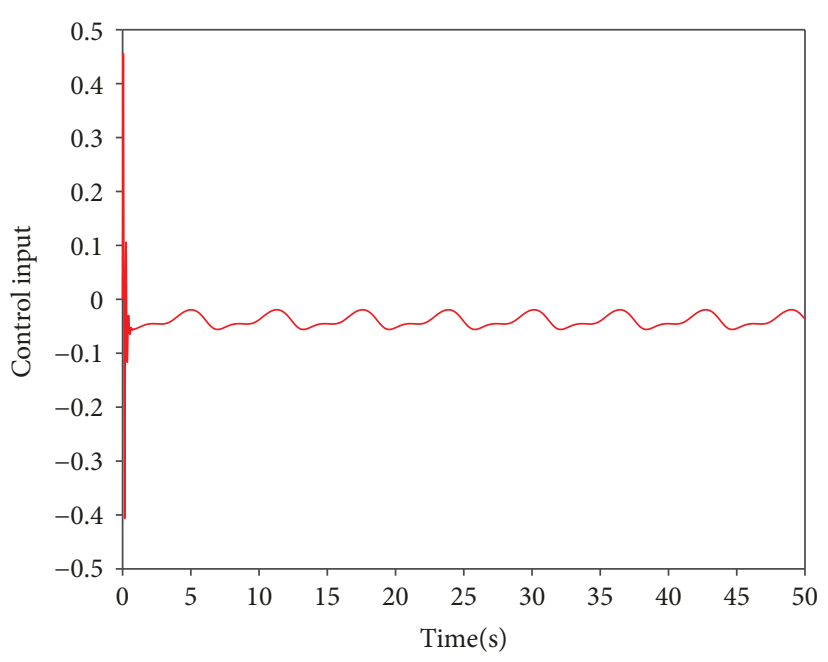

Figure 5: The control input of the proposed adaptive control for $u_{r}=(\pi / 6) \sin (t)$.
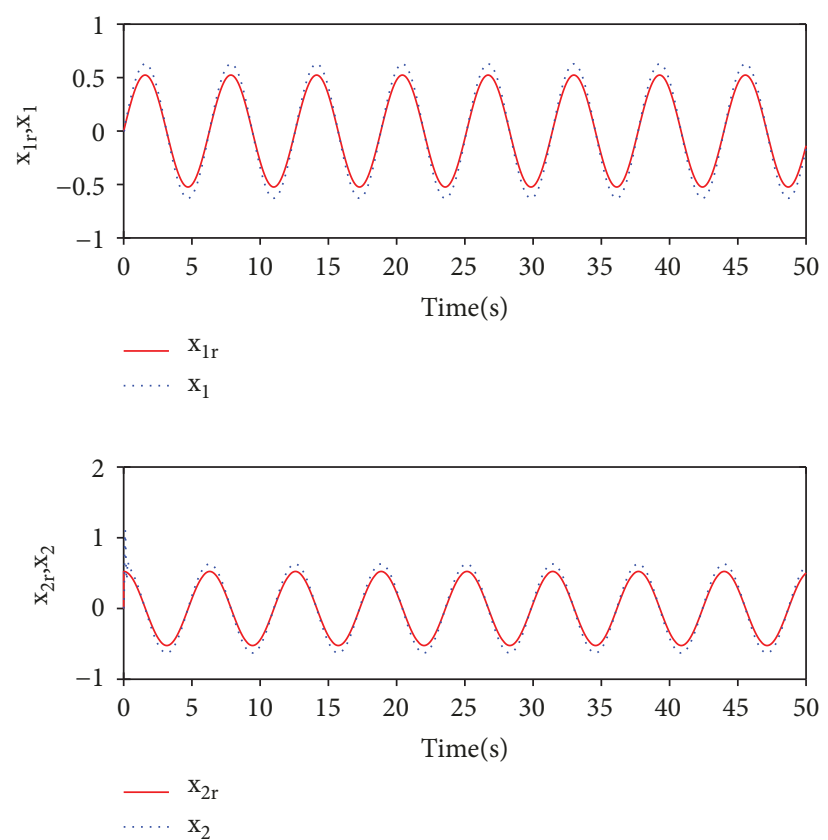

FIGURE 6: The adaptive control results of $x$ versus $x_{r}$ for $u_{r}=(\pi / 5)$ $\sin (t)$.

From Figure 3, it is clearly shown that the adaptive control results are well tracking the reference model for $u_{r}=(\pi / 6) \sin (t)$. The tracking errors are illustrated in Figure 4 and that demonstrates CNN identified the backlashlike hysteresis system, and the proposed approaches are effective. From these figures, we can find that oscillation is reduced at the beginning and this is due to the fact for the adaptive control.

We chose $u_{r}=(\pi / 5) \sin (t)$; the tracking results and errors are shown in Figures 6-8. With large amplitude of the sinusoid wave reference input, the tracking results are shown in Figure 6; the tracking errors are illustrated in Figure 7, and the control input is shown in Figure 8. 

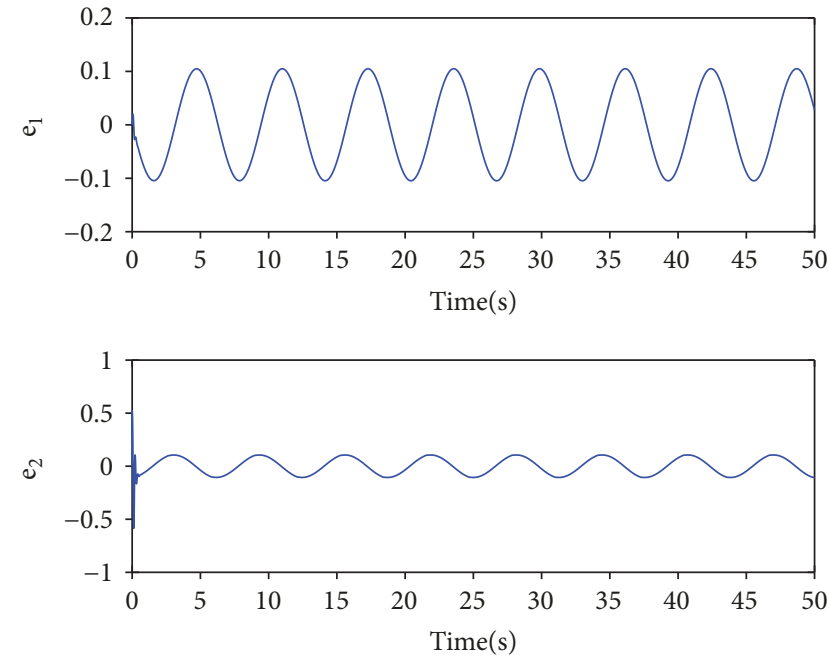

FIgURE 7: The errors of adaptive controller for $u_{r}=(\pi / 5) \sin (t)$.

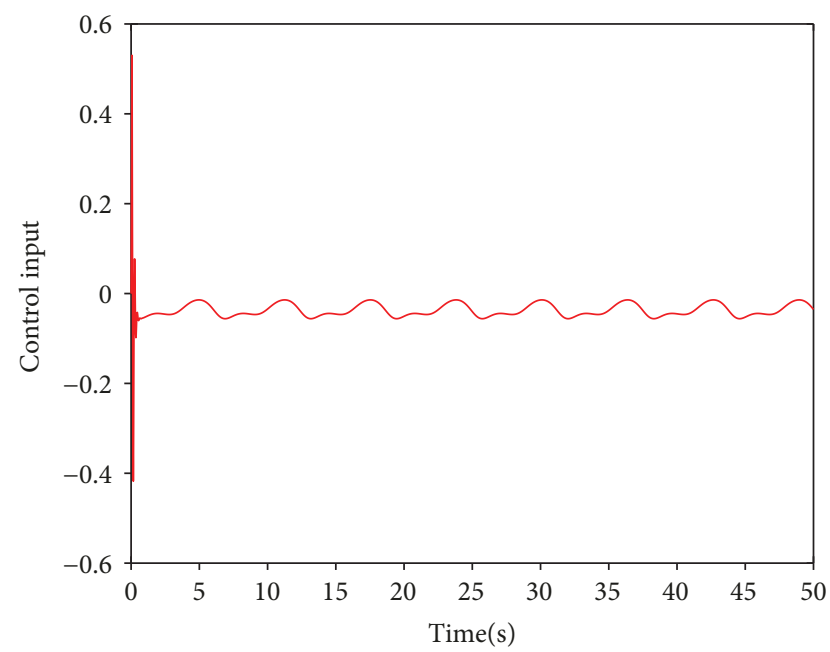

FIGURE 8: The control input of the proposed adaptive control for $u_{r}=(\pi / 5) \sin (t)$.

Compared with the results for $u_{r}=(\pi / 6) \sin (t)$, it is shown that the tracking precision is declined with large sinusoid wave amplitude. The same results are clearly shown in Figure 7, and the oscillation time is longer than the results of $u_{r}=(\pi / 6) \sin (t)$. It is declared that more sinal amplitude has more adjustment time and less control precision. This phenomenon is due to the fact and it verified the effectiveness of the proposed approaches.

In order to verify the effectiveness of the proposed adaptive $\mathrm{CNN}$ controller, we compare the proposed adaptive CNN controller, adaptive fuzzy controller [33-35], and PID controller in this paper. The system and parameters are chosen same as before, and the reference input is $u_{r}=(\pi / 6) \sin$ $(t)$. For PID controller, we chose $K_{p}=50, K_{i}=0.83, K_{d}=$ 0.6 , and the adaptive fuzzy controller similar reference [35] is used in this paper. The results are illustrated in Figures 9 and 10. It is clearly shown that the proposed adaptive CNN controller and adaptive fuzzy controller are better than the

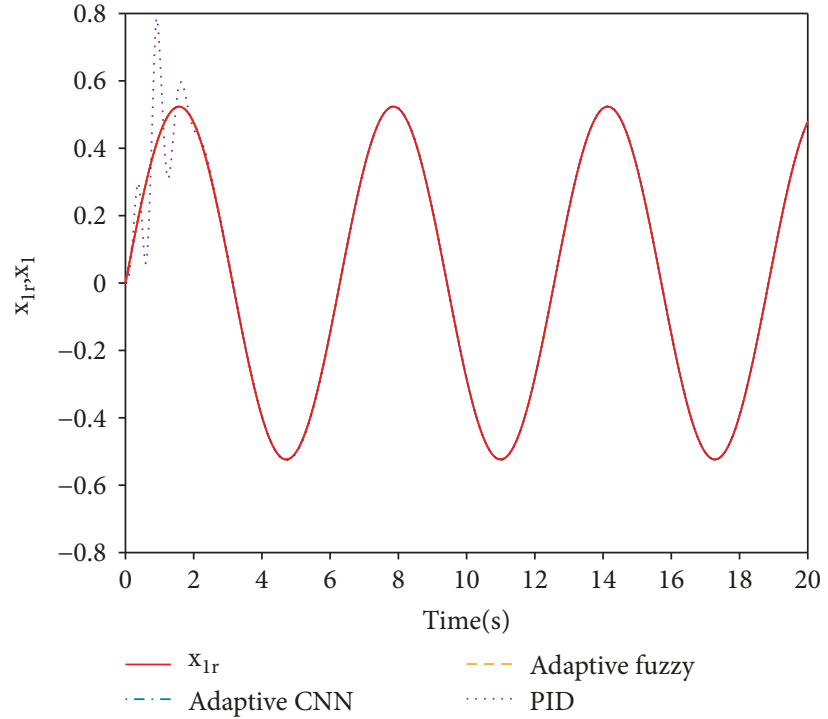

FIgURE 9: The comparison of the adaptive CNN, adaptive fuzzy, and PID controller for $x_{1}$.

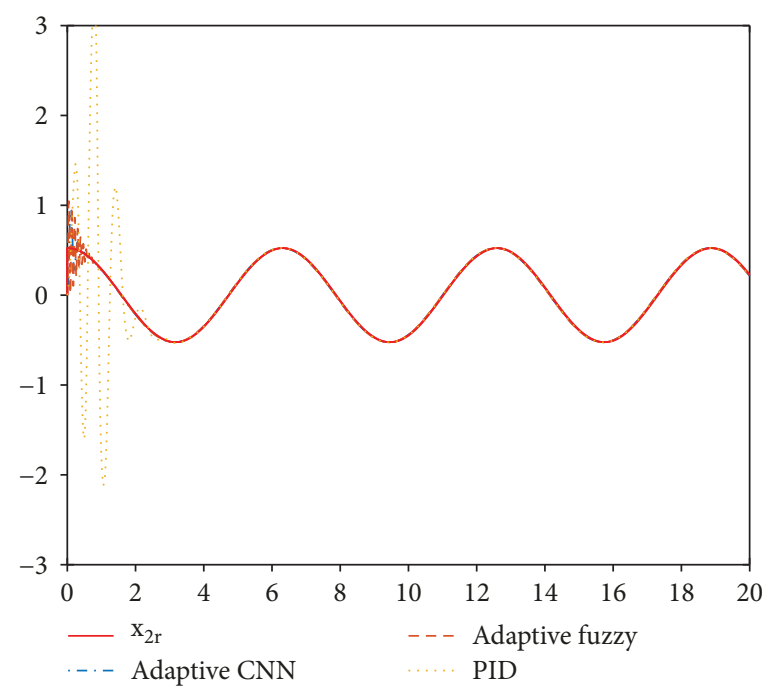

Figure 10: The comparison of the adaptive CNN, adaptive fuzzy, and PID controller for $x_{2}$.

PID controller. PID controller has longer dynamic procedure and more violent fluctuation. Adaptive CNN controller and adaptive fuzzy have similar performance in $x_{1}$, but adaptive CNN controller has more accuracy in dynamic procedure than adaptive fuzzy in $x_{2}$. It is illustrated that the proposed adaptive $\mathrm{CNN}$ controller has better performance for the hysteresis nonlinear system with backlash-like model.

\section{Conclusion}

This paper proposed a CNN multiscale identification and an adaptive control strategy to control the hysteresis nonlinearity where the hysteresis model was described by backlash-like model. The proposed multiscale $\mathrm{CNN}$ was firstly introduced approximating the backlash-like hysteresis 
system; a Lyapunov function guaranteed the effectiveness of the proposed identification method. Afterward, the tracking error was transformed into scalar error through Laplace and inverse Laplace transformation for simplifying the control design. Then, a new adaptive controller was designed with CNN based on the transformed error, and the Lyapunov theorem assured that all the signals included the tracking error in the closed-loop were UUB. Finally, simulations are designed that have verified the effectiveness of the proposed CNN identification and adaptive control.

\section{Data Availability}

The data used to support the findings of this study are available from the corresponding author upon request.

\section{Conflicts of Interest}

The authors declare that there are no conflicts of interest.

\section{Acknowledgments}

This work is supported by the Shandong Provincial Natural Science Foundation of China (ZR2017MF048), the National Natural Science Foundation of China (61433003), Shandong Science and Technology program of higher education (J17KA214), Scientific Research Foundation of Shandong University of Science and Technology for Recruited Talents (2016RCJJ035), Tai'an Science and Technology development program (2017GX0017).

\section{References}

[1] X. Gao, X. Ren, C. Zhu, and C. Zhang, "Identification and control for Hammerstein systems with hysteresis non-linearity," IET Control Theory \& Applications, vol. 9, no. 13, pp. 19351947, 2015.

[2] C.-Y. Su, Y. Stepanenko, J. Svoboda, and T. P. Leung, "Robust adaptive control of a class of nonlinear systems with unknown backlash-like hysteresis," IEEE Transactions on Automatic Control, vol. 45, no. 12, pp. 2427-2432, 2000.

[3] J. Zhou, C. Wen, and Y. Zhang, "Adaptive backstepping control of a class of uncertain nonlinear systems with unknown backlash-like hysteresis," IEEE Transactions on Automatic Control, vol. 49, no. 10, pp. 1751-1757, 2004.

[4] Y. Guo, J. Mao, and K. Zhou, "Rate-dependent modeling and $\mathrm{H}_{\infty}$ robust control of GMA based on Hammerstein model with Preisach operator," IEEE Transactions on Control Systems Technology, vol. 23, no. 6, pp. 2432-2439, 2015.

[5] X.-H. Gao, X.-M. Ren, F. Zheng, and Q.-Z. Wang, "Prescribed performance adaptive control for hysteresis hammerstein system," Beijing Ligong Daxue Xuebao/Transaction of Beijing Institute of Technology, vol. 36, no. 4, pp. 412-416, 2016.

[6] M. Al Janaideh, M. Rakotondrabe, and O. Aljanaideh, "Further results on hysteresis compensation of smart micropositioning systems with the inverse Prandtl-Ishlinskii compensator," IEEE Transactions on Control Systems Technology, vol. 24, no. 2, pp. 428-439, 2016.
[7] I. Ahmad, "Two degree-of-freedom robust digital controller design with Bouc-Wen hysteresis compensator for piezoelectric positioning stage," IEEE Access, vol. 6, pp. 17275-17283, 2018.

[8] Z. Liu, G. Lai, Y. Zhang, and C. L. P. Chen, "Adaptive neural output feedback control of output-constrained nonlinear systems with unknown output nonlinearity," IEEE Transactions on Neural Networks and Learning Systems, vol. 26, no. 8, pp. 1789-1802, 2015.

[9] H. J. Lee and J. J. Lee, "Modeling and time delay control of shape memory alloy actuators," Advanced Robotics, vol. 18, no. 9, pp. 881-903, 2004.

[10] D. Zhang, Y. Hu, and H. Guo, "Sliding model control based on Ackermann's formula for systems with backlash-like hysteresis," Jixie Gongcheng Xuebao/Chinese Journal of Mechanical Engineering, vol. 41, no. 1, pp. 11-15, 2005.

[11] R. Dong, Y. Tan, K. Janschek, and X. Chen, "Non-smooth predictive control for mechanical transmission systems with backlash-like hysteresis," Nonlinear Dynamics, vol. 85, no. 4, pp. 2277-2295, 2016.

[12] L. Liu, Z. Wang, Z. Huang, and H. Zhang, "Adaptive predefined performance control for MIMO systems with unknown direction via generalized fuzzy hyperbolic model," IEEE Transactions on Fuzzy Systems, vol. 25, no. 3, pp. 527-542, 2017.

[13] J. Na, X. Ren, and D. Zheng, “Adaptive control for nonlinear pure-feedback systems with high-order sliding mode observer," IEEE Transactions on Neural Networks and Learning Systems, vol. 24, no. 3, pp. 370-382, 2013.

[14] J. Na, A. S. Chen, G. Herrmann, R. Burke, and C. Brace, "Vehicle engine torque estimation via unknown input observer and adaptive parameter estimation," IEEE Transactions on Vehicular Technology, vol. 67, no. 1, pp. 409-422, 2018.

[15] J. Na, Y. Huang, X. Wu, G. Gao, G. Herrmann, and J. Z. Jiang, "Active adaptive estimation and control for vehicle suspensions with prescribed performance," IEEE Transactions on Control Systems Technology, pp. 1-15, 2017.

[16] S. Wang, X. Ren, J. Na, and T. Zeng, "Extended-state-observerbased funnel control for nonlinear servomechanisms with prescribed tracking performance," IEEE Transactions on Automation Science and Engineering, vol. 14, no. 1, pp. 98-108, 2017.

[17] S. Wang, J. Na, and X. Ren, "Rise-based asymptotic prescribed performance tracking control of nonlinear servo mechanisms," IEEE Transactions on Systems, Man, and Cybernetics: Systems, pp. 1-12, 2017.

[18] S. Wang, X. Ren, J. Na, and X. Gao, "Robust tracking and vibration suppression for nonlinear two-inertia system via modified dynamic surface control with error constraint," Neurocomputing, vol. 203, pp. 73-85, 2016.

[19] R. Sun, J. Na, and B. Zhu, "Robust approximation-free prescribed performance control for nonlinear systems and its application," International Journal of Systems Science, vol. 49, no. 3, pp. 511-522, 2018.

[20] J. Na, G. Herrmann, and K. Zhang, "Improving transient performance of adaptive control via a modified reference model and novel adaptation," International Journal of Robust and Nonlinear Control, vol. 27, no. 8, pp. 1351-1372, 2017.

[21] C.-H. Lin, "PMSM servo drive for V-belt continuously variable transmission system using hybrid recurrent Chebyshev NN control system," Journal of Electrical Engineering and Technology, vol. 10, no. 1, pp. 408-421, 2015.

[22] G. Sun, X. Ren, and D. Li, "Neural active disturbance rejection output control of multimotor servomechanism," IEEE 
Transactions on Control Systems Technology, vol. 23, no. 2, pp. 746-753, 2015.

[23] A.-M. Zou, K. D. Kumar, Z.-G. Hou, and X. Liu, "Finite-time attitude tracking control for spacecraft using terminal sliding mode and Chebyshev neural network," IEEE Transactions on Systems, Man, and Cybernetics, Part B (Cybernetics), vol. 41, no. 4, pp. 950-963, 2011.

[24] Q. Chen, L. Shi, J. Na, X. Ren, and Y. Nan, “Adaptive echo state network control for a class of pure-feedback systems with input and output constraints," Neurocomputing, vol. 275, pp. 1370-1382, 2018.

[25] J. C. Patra and A. C. Kot, "Nonlinear dynamic system identification using Chebyshev functional link artificial neural networks," IEEE Transactions on Systems, Man and Cybernetics, Part B (Cybernetics), vol. 32, no. 4, pp. 505-511, 2002.

[26] Y. Huang, J. Na, X. Wu, and G. Gao, "Approximation-free control for vehicle active suspensions with hydraulic actuator," IEEE Transactions on Industrial Electronics, vol. 65, no. 9, pp. 7258-7267, 2018.

[27] C. Yang, Y. Jiang, W. He, J. Na, Z. Li, and B. Xu, “Adaptive parameter estimation and control design for robot manipulators with finite-time convergence," IEEE Transactions on Industrial Electronics, vol. 65, no. 10, pp. 8112-8123, 2018.

[28] Y. Huang, J. Na, X. Wu, G.-B. Gao, and Y. Guo, "Robust adaptive control for vehicle active suspension systems with uncertain dynamics," Transactions of the Institute of Measurement and Control, vol. 40, no. 4, pp. 1237-1249, 2018.

[29] Q. Chen, X. Ren, J. Na, and D. Zheng, “Adaptive robust finitetime neural control of uncertain PMSM servo system with nonlinear dead zone," Neural Computing and Applications, vol. 28, no. 12, pp. 3725-3736, 2017.

[30] D. D. Zheng, W. F. Xie, T. Chai, and Z. Fu, "Identification and trajectory tracking control of nonlinear singularly perturbed systems," IEEE Transactions on Industrial Electronics, vol. 64, no. 5, pp. 3737-3747, 2017.

[31] D. Zheng, W. F. Xie, X. Ren, and J. Na, "Identification and control for singularly perturbed systems using multitime-scale neural networks," IEEE Transactions on Neural Networks and Learning Systems, vol. 28, no. 2, pp. 321-333, 2017.

[32] A. M. Annaswamy, F. P. Skantze, and A.-P. Loh, “Adaptive control of continuous time systems with convex/concave parametrization,” Automatica, vol. 34, no. 1, pp. 33-49, 1998.

[33] H. A. Yousef, M. Hamdy, and K. Nashed, " $\mathscr{L}_{1}$ adaptive fuzzy controller for a class of nonlinear systems with unknown backlash-like hysteresis," International Journal of Systems Science, vol. 48, no. 12, pp. 2522-2533, 2017.

[34] Y. Li, S. Tong, and T. Li, “Adaptive fuzzy output feedback control of nonlinear uncertain systems with unknown backlashlike hysteresis based on modular design," Neural Computing and Applications, vol. 23, Supplement 1, pp. 261-270, 2013.

[35] S. Wang, X. Ren, and J. Na, "Adaptive dynamic surface control based on fuzzy disturbance observer for drive system with elastic coupling," Journal of the Franklin Institute, vol. 353, no. 8, pp. 1899-1919, 2016. 


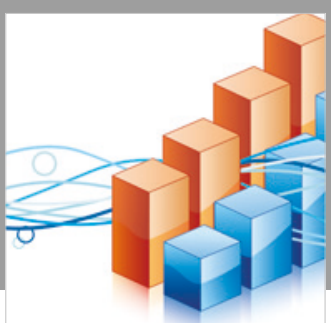

Advances in

Operations Research

\section{-n-m}
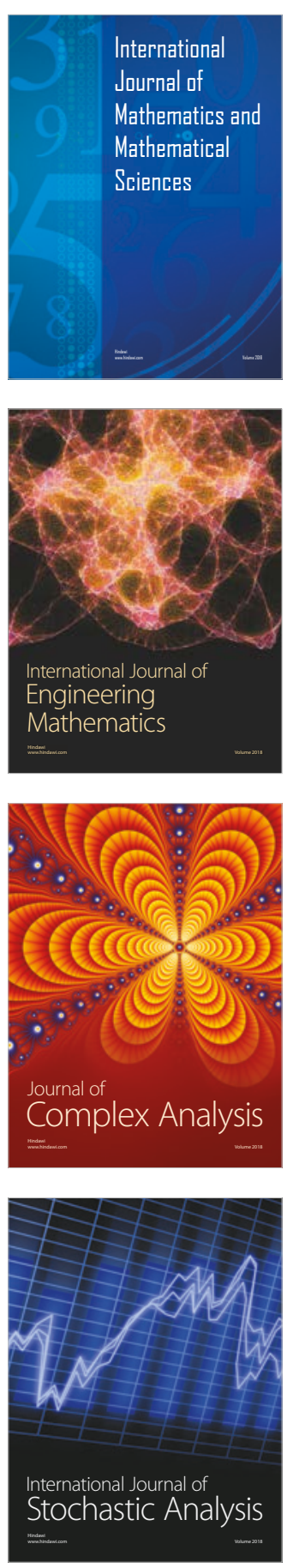
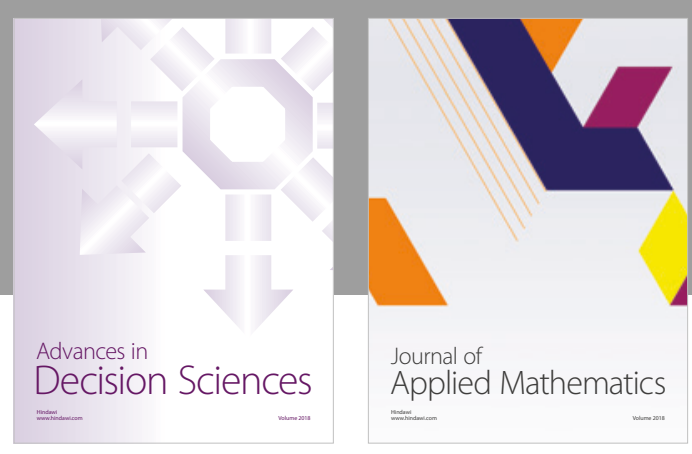

Journal of

Applied Mathematics
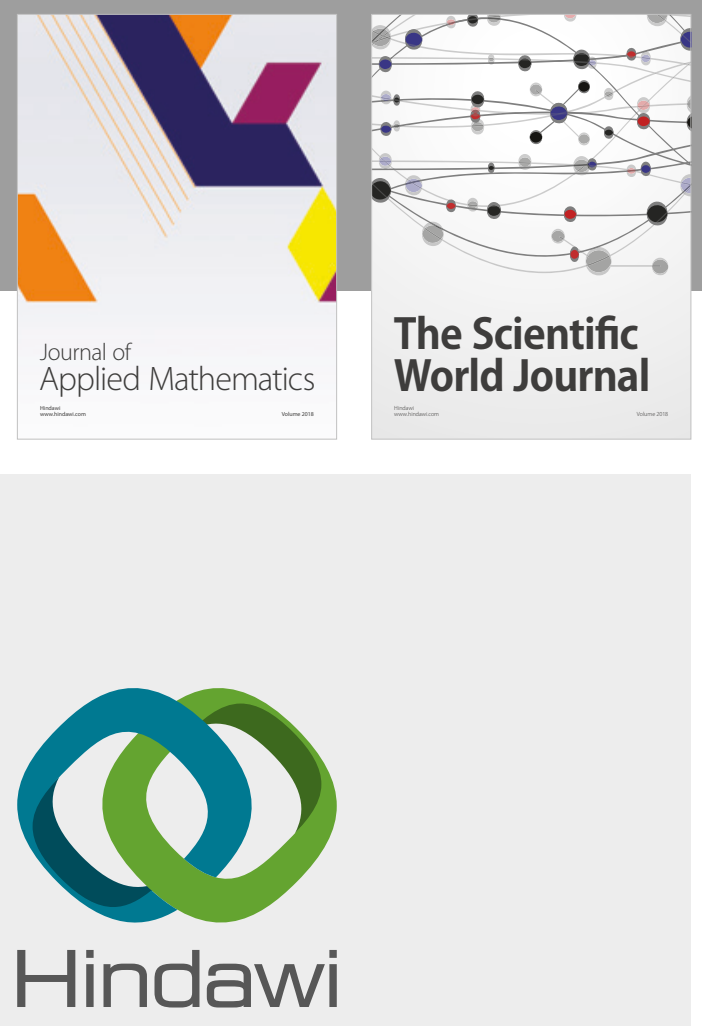

Submit your manuscripts at

www.hindawi.com

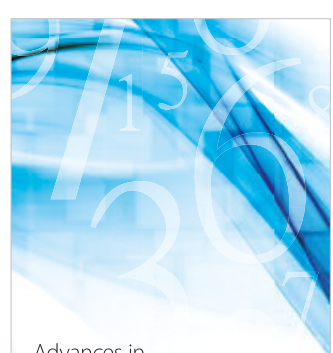

Advances in
Numerical Analysis
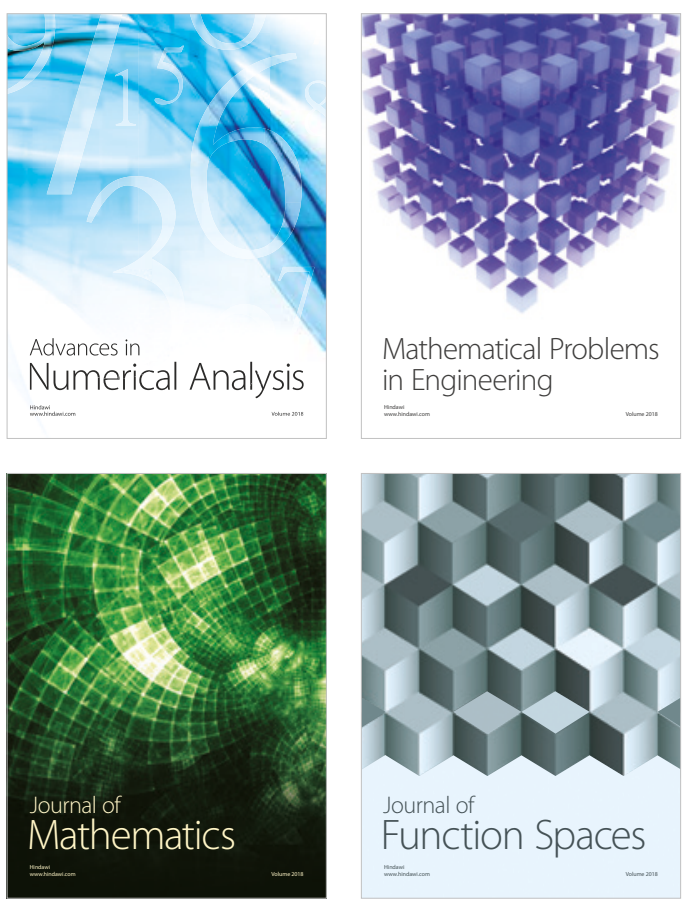

Mathematical Problems in Engineering

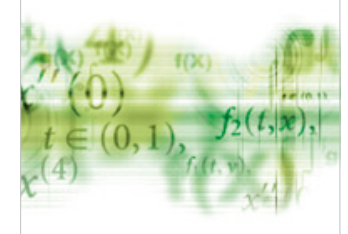

International Journal of

Differential Equations

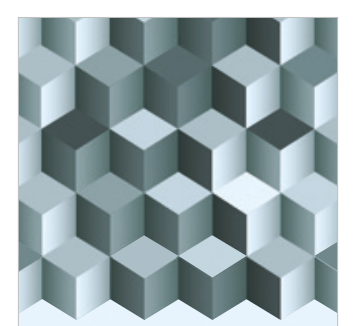

Journal of

Function Spaces
The Scientific

World Journal

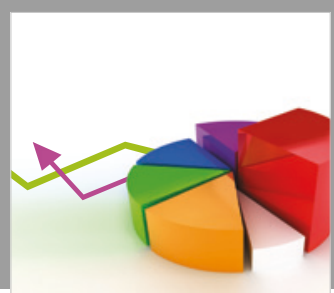

Journal of

Probability and Statistics
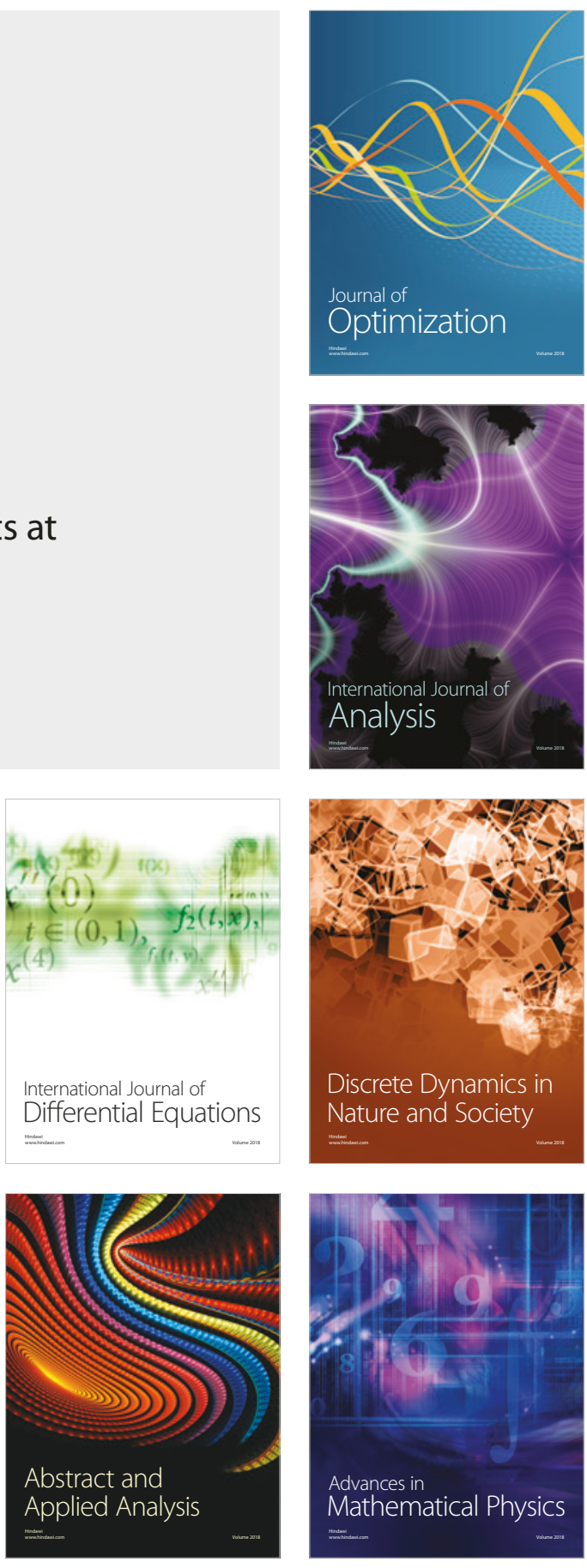